\title{
Selected filmography and videography
}

Abbreviations: Prod. $=$ production; Dist. $=$ distribution.

Alphaville (1965) Directed by Jean-Luc Godard. London, Connoisseur Video [Video: VHS].

Anthology: George Barber (1997) London, London Electronic Arts [Video: VHS].

Anthology: John Smith Vols 1 \& 2 (1997) London, London Electronic Arts [Video: VHS].

Associations (1975) Directed by John Smith. Prod. London, John Smith/

Royal College of Art. Dist. Lux [Film: 16mm] Available on the compilation Anthology: John Smith. Volume 1. (1997) London, London Electronic Arts [Video: VHS].

At the Fountainhead (of German Strength) (1980) Directed by Anthea Kennedy \& Nick Burton. Prod. and Dist. London, British Film Institute [Film: 16mm].

Birdman (1975) Directed by Anthea Kennedy \& Nick Burton. Prod. London, World Service/Royal College of Art. Dist. Anthea Kennedy [Film: 16mm]. Birds, The (1963) Directed by Alfred Hitchcock. Hollywood, Universal Pictures. [Video: DVD].

Black Tower, The (1985-87) Directed by John Smith. Prod. London, Arts Council of Great Britain. Dist. Lux [Film: 16mm]. Available on the compilation Anthology: John Smith. Volume 1.(1997) London, London Electronic Arts [Video: VHS].

Blue Lagoon, The (1980) Directed by Randal Kleiser. Culver City, Columbia Pictures Corporation [Film: $35 \mathrm{~mm}$ ].

Blue Monday (1984) Directed by The Duvet Brothers. Prod. London, The Duvet Brothers. Dist. Lux [Video]. Available on the compilation The Greatest Hits of Scratch Volume One (1985) Directed by George Barber. London, Scratch Video [Video: VHS].

Bucks Fizz (1984) Directed by Jeffrey Hinton [Video]. Available on the compilation The Greatest Hits of Scratch Video Volume One (1985) Directed by George Barber. London, Scratch Video [Video: VHS].

Casablanca (1942) Directed by Michael Curtiz. Burbank, Warner Bros. [Film: 35mm]. 
Catalog (1961) Directed by John Whitney. Prod. Motion Graphics Inc. Dist. BFI [as part of the Whitney Brothers Programme] [Film: $16 \mathrm{~mm}]$.

Central (2001) Directed by Scott Rankin. Prod. Hong Kong, Videotage [video]. Dist. Scott Rankin [Video: DVD].

Chelsea Girls, The (1966) Directed by Paul Morrisey \& Andy Warhol. New York, The Film-makers' Cooperative [Film: $16 \mathrm{~mm}$ ].

Crack-up (1946) Directed by Irving Reis. Atlanta, Turner Home Entertainment [Video: VHS].

Deep, The (1977) Directed by Peter Yates. Los Angeles, Casablanca Filmworks/EMI Films [Film: 35mm].

Deviant Beauty (1996) Directed by Tina Keane. Prod. London, London Production Fund [video]. Dist. Lux [Video: VHS].

Diagonal Symphony (1925) Directed by Viking Eggeling [Film: 35mm]. Available on the compilation Experimental Cinema of the 1920s and '30s. (Films from the Raymond Rohauer Collection). New York, Kino Video [Video: DVD].

Doodlin': Impressions of Len Lye (1987) Directed by Keith Griffiths. London, Illuminations for Channel 4/ESTV New Zealand [Video: VHS].

Fantasia (1940) Produced by Walt Disney. Burbank, Walt Disney Pictures [Film: $35 \mathrm{~mm}$ ].

Fast and Furry-ous (1949) Directed by Chuck Jones. Available on the compilation Looney Tunes Golden Collection. Burbank, Warner Home Video [Video: DVD].

Felix the Cat in Astronomeows (1928) Directed by Otto Messmer. New York, Pat Sullivan Films [Film: 35mm] Available on the compilation Felix the Cat in Bold King Cole. Diamond Films [Video: VHS].

Five Film Exercises (1943-44) Produced by John and James Whitney. Prod. Hollywood, John and James Whitney [Film: 16mm] Dist. Lux [Film: $16 \mathrm{~mm}]$.

Forbidden Planet (1956) Directed by Fred M. Wilcox. Taipei, Great Music [Video: DVD].

Gone With the Wind (1939) Directed by Victor Flemming. Los Angeles, Selznick International Pictures [Film: 35mm].

The Greatest Hits of Scratch Video Volume One (1985) Directed by George Barber. Volume Two (1986) Produced by George Barber, The Duvet Brothers, Tim Morrison. London, Scratch Video [Video: VHS].

Holiday Inn (1942) Directed by Mark Sandrich. Hollywood, Paramount Pictures Inc. [Film: 35mm].

Il Deserto Rosso (1964) Directed by Michelangelo Antonioni. London, Connoisseur Video [Video: VHS].

Informer, The (1935) Directed by John Ford. Hollywood, RKO Radio Pictures Inc. [Film: 35mm].

King Kong (1933) Directed by Merian C. Cooper \& Ernest B. Schoedsack. Hollywood, RKO Radio Pictures Inc. [Film: 35mm]. 
Lapis (1963-66) Produced by James Whitney, Hollywood, [Film: 16mm]. Dist. London, Lux [Film: $16 \mathrm{~mm}$ ].

Last Days, The (1998) Directed by James Moll. Los Angeles, Survivors of the Shoah Visual History Foundation/Ken Lipper-June Beallor [Film: $35 \mathrm{~mm}$.

L'avventura (1960) Directed by Michelangelo Antonioni. London, Connoisseur Video [Video: VHS].

L'eclisse (1962) Directed by Michelangelo Antonioni. New York, Criterion Collection [Video: DVD].

M. Hulot's Holiday (1953) Directed by Jacques Tati. London, Connoiseur Video [Video: VHS].

My Favourite Wife (1940) Directed by Garson Kanin. Watford, Herts., Cinema Club [Video: VHS].

Neighbours (1952) Directed by Norman McLaren. Available on the compilation Norman McLaren: Selected Films. London, Connoisseur Video [Video: VHS].

Night of a 1000 Eyes (1984) Directed by Kim Flitcroft and Sandra Goldbacher. Dist. Lux [Video]. Available on the compilation The Greatest Hits of Scratch Volume One (1985) Directed by George Barber. London. London: Scratch Video [Video: VHS].

Om (1986) Directed by John Smith. Prod. London, John Smith. Dist. Lux [Film: 16mm]. Available on the compilation Anthology: John Smith. Volume 1. (1997) London, London Electronic Arts [Video: VHS].

On the Waterfront (1954) Directed by Elia Kazan. Culver City, Columbia Pictures Corporation/ Horizon Pictures [Film: $35 \mathrm{~mm}$ ].

Oskar Fischinger: Visual Music (1992) Directed by Keith Griffiths. London, Koninck International [Video: VHS].

Passing, The (1991) Directed by Bill Viola. Amsterdam, Éditions à voir [Video: DVD].

Path (2003) Directed by Scott Rankin. Prod. Normal, IL., Scott Rankin. Dist. Video Data Bank [Video: DVD].

Peace and Plenty (1939) Directed by Ivor Montagu. Prod. London, Kino. Featured in the compilation Workers films of the 1930s (1981) Directed by Victoria Wegg-Prosser. London, BFI [Film: 16mm].

Piccadilly (2004) Directed by Scott Rankin. Prod. \& Dist. Normal, IL., Scott Rankin. [Video: DVD].

President's World, The (1985) Directed by Gorilla Tapes. Dist. Lux [Video]. Available on the compilation The Greatest Hits of Scratch Video Volume Two (1986) Produced by George Barber, The Duvet Brothers, Tim Morrison. London, Scratch Video [Video: VHS].

Psycho (1960) Directed by Alfred Hitchcock. Hollywood, Paramount Pictures [Film: 35mm].

Pull My Daisy (1959) Directed by Robert Frank and Alfred Leslie. New York, G-String Enterprises [Film: 35mm]. 
Pure, The (1993) Directed by Scott Rankin. Prod. Chicago, Center for New Television. Dist. Video Data Bank [Video: DVD].

Reichstag Fire Part I, The (1976) Directed by Anthea Kennedy and Nick Burton. Prod. London, World Service/Royal College of Art. Dist. Anthea Kennedy [Film: 16mm].

Searchers, The (1956) Directed by John Ford. Burbank, Warner Bros. [Film: 35mm].

Shaft (1971) Directed by Gordon Parks. Los Angeles, MGM/Shaft Productions [Film: $35 \mathrm{~mm}$ ].

Spellbound (1945) Directed by Alfred Hitchcock. London, Pearson Television International [Video: DVD].

Swing, The (1978) Directed by Tina Keane. Prod. London, Tina Keane.

Dist. Lux [Video: VHS].

Synchromy (1971) Directed by Norman McLaren. Montreal, National Film Board of Canada [Film: $35 \mathrm{~mm}$ ] available on the compilation Norman McLaren: Selected Films (2000) London, Connoisseur Video [Video: VHS]. Take It (1984) Directed by The Duvet Brothers. Prod. London, The Duvet Brothers [Video]. Available on the compilation The Greatest Hits of Scratch Video Volume One (1985) Directed by George Barber. London, Scratch Video [Video: VHS].

Thin Red Line, The (1998) Directed by Terence Malick. Los Angeles, Twentieth Century Fox Home Entertainment [Film: DVD].

This and That (Part Two) (1990) Directed by Scott Rankin. Prod. Chicago, Centre for New Television/ National Endowment for the Arts/New York, Art Matters Inc. Dist. Scott Rankin [Video: VHS].

T.V. of Tomorrow (1953) Directed by Tex Avery. Los Angeles, MGM [Film: $35 \mathrm{~mm}]$.

Vivre sa vie (1962) Directed by Jean-Luc Godard. Hereford, Nouveaux Pictures [Video: DVD].

War Machine (1984) Directed by The Duvet Brothers. Prod. London, The Duvet Brothers. Dist. Lux [Video]. Available on the compilation The Greatest Hits of Scratch Video Volume One (1985) Directed by George Barber. London, Scratch Video [Video: VHS].

Wavelength (1967) Directed by Michael Snow. New York, Michael Snow [Film: 16mm]. Dist. London, BFI [Film: 16mm].

Way Out West (1937) Directed by James W. Horne. Culver City, Hal Roach Studios/Stan Laurel Productions [Film: $35 \mathrm{~mm}$ ].

What's Opera Doc? (1957) Directed by Chuck Jones. Burbank, Warner Bros. [Film: 35mm].

White Christmas (1954) Michael Curtiz. Hollywood, Paramount Pictures [Film: 35mm].

White Zombie (1932) Directed by Victor Halperin. Elstree, Herts, Elstree Hill Entertainment [Video: DVD].

Yantra (1950-58) Directed by James Whitney. Prod. Uroboros Films. Dist. London, Lux [Film: 16mm]. 
Yes Frank, No Smoke (1985) Directed by George Barber. Dist. Lux [Video]. Available on the compilation Anthology: George Barber (1997) London, London Electronic Arts [Video: VHS]. 